\section{A Switch Therapy Protocol with Intravenous Azithromycin and Ciprofloxacin Combination for Severe, Relapsing Chronic Bacterial Prostatitis: a Prospective Non-comparative Pilot Study}

\author{
Adela Kolumbić Lakošs ${ }^{1}$, Višnja Škerk ${ }^{2}$, Gordan Maleković ${ }^{1}$, Tatjana Dujnić \\ Špoljarević ${ }^{1}$, Dražen Kovačić ${ }^{1}$, Miram Pasini ${ }^{2}$, Alemka Markotić ${ }^{2}$, Vittorio Magri ${ }^{3}$, \\ Gianpaolo Perletti ${ }^{4}$ \\ ${ }^{1}$ Pliva Croatia Ltd. \\ 2University Hospital for Infectious Diseases "Dr. Fran Mihaljević", Zagreb, Croatia \\ ${ }^{3}$ Urology and Sonography Secondary Care Clinic, Azienda Ospedaliera Istituti Clinici di \\ Perfezionamento, Milano, Italy \\ ${ }^{4}$ Laboratory of Toxicology and Pharmacology, Department of Biomedical Inf./Env./ \\ Comm. Sciences, Università degli Studi dell'Insubria, Busto A./Varese, Italy
}

Summary chronic bacterial prostatitis (CBP) is characterized by intense clinical symptoms, frequent relapse episodes and poor quality of life. Aggressive antibacterial therapy is warranted to eradicate causative pathogens and to achieve a permanent cure. We administered a "switch-therapy" protocol to 30 patients showing severe CBP symptoms and two or more relapse episodes in the previous 12 months. Patients received intravenous azithromycin (500 mg/day) and ciprofloxacin (800 mg/day) for three days, followed by oral ciprofloxacin (1 g/day) for 25 days. Twenty-seven (90\%) patients showed pathogen eradication at test-of-cure (TOC) visit. Five cases of infection relapse were detected at follow-up. At the TOC visit,
25 patients (83\%) showed mild/absent symptoms, measured with the NIH-chronic prostatitis symptom index. These results indicate the efficacy of a "switch-therapy" protocol, based on combined azithromycin and ciprofloxacin. Comparative studies on larger CBP patient populations are warranted to confirm these encouraging results.

Key words: chronic prostatitis, chronic bacterial prostatitis, chronic pelvic pain syndrome, macrolide, fluoroquinolone, genitourinary infections, azithromycin, ciprofloxacin, NIH-CPSI

\section{Introduction}

Chronic prostatitis syndromes are serious, invaliding conditions. The quality of life for a man suffering from chronic prostatitis is similar to that experienced by patients affected by myocardial infarction, unstable angina, or Crohn's disease. $^{1}$

Symptomatic chronic prostatitis syndromes are generally classified as bacterial or abacterial. Category II chronic bacterial prostatitis (CBP, National Institutes of Health $[\mathrm{NIH}]$ classification) is diagnosed when uropathogens are cultured from a chronically infected prostate in patients suffering from repeated symptomatic episodes of bacteriuria. $^{2}$

CBP signs and symptoms may include perineal pain, testicular pain and/or other forms of pelvic pain, irritative and/ or obstructive bladder symptoms, hematuria, pyuria, ejaculatory pain and sexual dysfunction. CBP lacks the typi- cal systemic hallmarks of acute prostatitis, such as fever, chills, malaise and other symptoms. ${ }^{2,3}$

When severe symptoms are present in CBP patients, aggressive, long-term antibacterial therapy is warranted to eradicate causative pathogens, to attenuate the intense symptoms, and to achieve a permanent cure..$^{3,4}$ Fluoroquinolones (FQs) are recommended as first-choice antibacterial agents for treatment of category II CBP. International guidelines indicate a 4-12 week course of ciprofloxacin, levofloxacin, norfloxacin or other FQs for treatment of infection caused by susceptible pathogens. ${ }^{5,3}$

FQ therapy can achieve pathogen eradication in over $80 \%$ of cases. ${ }^{6}$ However, success of antibacterial therapy is often transient. Relapse occurs frequently, and is caused in most cases by incomplete pathogen eradication, probably due to insufficient distribution of antibiotics to the prostate, but also possibly due to survival of pathogens in the form of bacterial chemoresistant biofilms. ${ }^{7}$ CBP symptoms 
and infection relapses may continue to flare up for months or years, with a considerable impact on the quality of life of patients. Current research in the field of chronic bacterial prostatitis focuses on the optimization of therapeutic protocols aimed at achieving higher eradication rates and at minimizing the probability of relapse. ${ }^{8}$ Over the last few years, we have optimized an aggressive antibacterial protocol for CBP, based on the administration of oral ciprofloxacin combined with oral azithromycin for 4 to 6 weeks. This regimen was well tolerated and was characterized by high eradication rates, by rapid and sustained symptom relief, and by a low number of recurrences. ${ }^{9-13}$ In order to improve the outcome of antibacterial therapy in specific cases of CBP characterized by severe clinical symptoms concomitant with frequent infection relapses, we designed a therapeutic protocol aimed at exploiting (i) the effectiveness of the combination of antibacterial fluoroquinolones and macrolides, (ii) the important non-antibiotic intrinsic properties of macrolides, as well as (iii) the complete bioavailability of these antibacterial agents and the optimal systemic drug exposure achievable with intravenous drug administration.

Because long-term intravenous treatment is not sustainable in CBP patients, a "switch-therapy" protocol was designed to ensure optimal patient compliance.

A pilot study was conducted to investigate the safety and the clinical/microbiological efficacy of this therapeutic protocol.

\section{Patients and methods}

This study was conducted at the Outpatient Department for Urogenital Infections, University Hospital for Infectious Diseases “Dr. Fran Mihaljević”, Zagreb, Croatia, between 2008 and 2010. The Ethics Committee of the Hospital approved the study. An informed consent was signed by all patients after counselling.

\section{Inclusion/exclusion criteria}

We enrolled patients older than 20 years of age with CBP caused by traditional uropathogens and no evidence of anatomical abnormality of the genitourinary tract, assessed by prostate ultrasound imaging.

Patients were included in this study if they manifested the signs and symptoms of category II CBP, according to the $\mathrm{NIH}$ criteria (National Institute of Diabetes and Digestive and Kidney Diseases Chronic Prostatitis Workshop, 1995).

Signs/symptoms included pain or discomfort in the lower back and in the perineal, penile, suprapubic, scrotal or groin areas, painful ejaculation, dysuria, urinary frequency, urgency, hesitancy, nycturia, urinary retention or decreased urinary stream, prostate asymmetry or tenderness.

Specific inclusion criteria for this study were: (i) severe clinical symptoms, defined as the sum of $\mathrm{NIH}$-Chronic Pros- equal or superior to $17,{ }^{14}$ and (ii) two or more relapse episodes of CBP occurring in the previous 12 months.

CBP patients were excluded from the study if they presented any of the following conditions: category I acute bacterial prostatitis, chronic prostatitis involving Chlamydia trachomatis, Ureaplasma urealyticum, fungi or protozoa, category III chronic prostatitis/chronic pelvic pain syndrome, therapy with antibacterial agents or other medications effective at the prostatic level within a period of 14 days prior to prostatitis diagnosis, renal/hepatic/cardiac insufficiency (for liver disease: AST/ALT levels twice above the upper limit were exclusion criteria), arrhythmia, long QT syndrome, indwelling catheters, cystostomy, ureterostomy, previous prostatic surgery or radiotherapy, neoplasia, chronic diarrheal disease or any other gastrointestinal condition affecting drug absorption.

\section{Diagnosis}

The following data were obtained for each patient: clinical history, clinical status (including digitorectal examination) and the severity of chronic prostatitis symptoms, scored with a Croatian translation of the NIH-CPSI questionnaire, rating pain, voiding symptoms and the impact of the disease on the quality of life of patients.

Urethral swab specimens and samples of urine and expressed prostatic secretions (EPS), according to the "4-glass" lower urinary tract segmented test ${ }^{15}$ were collected in each patient. Quantitative segmented cultures and bacterial identification of Gram-negative and Gram-positive bacteria in first-voided, midstream, and post-massage voided urine $\left(\mathrm{VB}_{3}\right)$ specimens and EPS were preformed at the Clinical Microbiology Laboratory of the University Hospital for Infectious Diseases “Dr Fran Mihaljević”, Zagreb, by standard microbiological techniques.

The number of leukocytes and Gram-positive and Gramnegative bacteria was determined in $1 \mathrm{ml}$ of urine from the segmented test. Expressed prostatic secretions (EPS) and urine samples collected immediately after prostatic massage were also examined for the presence of Chlamydia trachomatis, Ureaplasma urealyticum, Mycoplasma hominis and Trichomonas vaginalis. Urethral swab specimens were analyzed for Chlamydia trachomatis, Ureaplasma urealyticum and Mycoplasma hominis.

\section{Study design and treatment regimen}

This prospective, noncomparative open-label pilot study was performed on a cohort of $30 \mathrm{CBP}$ patients selected on the basis of stringent inclusion/exclusion criteria. All patients received a 4-week "switch-therapy" antibacterial protocol (post-hoc assessed compliance: 100\%), based on the administration of intravenous azithromycin (500 mg once daily) and intravenous ciprofloxacin (400 mg twice daily) for three days, followed by oral ciprofloxacin (500 mg twice daily) for 25 days.

During the course of the study patients received no additional medication that would relieve their symptoms, or 
would act as a confounder during evaluation of the clinical endpoints of the study. Clinical and microbiological workup was performed 4-6 weeks (test-of-cure [TOC] visit) and 6 months (Follow-up [FU] visit) after the last ciprofloxacin dose.

\section{Clinical efficacy endpoints and}

\section{microbiological response definitions}

Microbiological efficacy was the primary outcome of this study. The pathogen loads in expressed prostatic secretions (EPS) or in bladder urine specimens collected immediately after prostatic massage $\left(\mathrm{VB}_{3}\right)$ were microbiological outcome measures. A bacterial count inferior to $10^{3}$ $\mathrm{CFU} / \mathrm{ml}$ in EPS or $\mathrm{VB}_{3}$ defined microbiological eradication. If only Gram-positive cocci were found (with the exception of Enterococci and Staphylococcus aureus), a bacterial count inferior to $10^{4} \mathrm{CFU} / \mathrm{ml}$ was the eradication criterion. Microbiological persistence was defined as bacterial counts of causative pathogen equal or superior to eradication thresholds. Eradication with superinfection was defined as baseline pathogen eradicated $\left(<10^{3} \mathrm{CFU} /\right.$ $\mathrm{ml}$ or $<10^{4} \mathrm{CFU} / \mathrm{ml}$ if Gram-positive cocci, with the exception of Enterococci and S. aureus) with appearance of a new pathogen $\left(\geq 10^{3} \mathrm{CFU} / \mathrm{ml}\right.$ or $\geq 10^{4} \mathrm{CFU} / \mathrm{ml}$ if Gram-positive cocci).

Clinical efficacy, the secondary outcome of the study, was measured using the NIH-CPSI. The clinical efficacy endpoint was the sum of $\mathrm{NIH}-\mathrm{CPSI}$ pain and voiding symptom scores, categorized into mild (0 to 9 points), moderate (10 to 16 points) and severe (17 to 31 points) according to McNaughton Collins et al. ${ }^{14}$ Statistical significance of decreases in NIH-CPSI total scores, pain + voiding scores or quality of life impact scores was also calculated by nonparametric analysis. Tolerability of the experimental pro- tocol as well as possible adverse events were evaluated at the TOC visit, 6 months after completion of therapy and whenever adverse effects were spontaneously reported by patients.

\section{Sample size and statistical methods}

The Wilcoxon signed-rank test (WSRT) was used to analyze intragroup differences in NIH-CPSI scores at different time points, with an $\alpha$ error of $5 \%$ to define significance. Onetailed analysis was used, as it was only feasible that symptoms of untreated bacterial prostatitis patients would attenuate upon aggressive antibacterial therapy.

A priori sample size computation was performed, based on a minimally acceptable estimated effect size of -0.91 , corresponding to a "slight symptom improvement" scored with the NIH-CPSI test, according to Propert et al. ${ }^{16} \mathrm{~A}$ sample size of 10 was adequate for a one-tailed analysis, with a 5\% $\alpha$ error probability and $80 \% 1-\beta$ error probability.

The XLStatistics 5.71 program (http://www.deakin.edu.

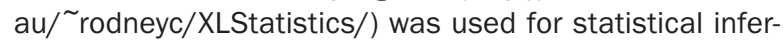
ence; the G*Power 3.1 software ${ }^{17}$ was used for a priori and post-hoc statistical power analysis.

\section{Results}

The median age of enrolled patients was 51 (range: 27$65)$. At the baseline visit, 27 and 3 patients manifested single-pathogen and double-pathogen infections, respectively. The baseline median NIH-CPSI total score of the patient population was 32 (range: 25-38).

Table 1 shows the microbiological presentation of patients at the end of treatment (test-of-cure visit) and at follow-up. Twenty-seven patients (90\%) showed eradication of caus-

Table 1. Microorganisms isolated from prostate-specific specimens and per-pathogen eradication data in 30 CBP patients subjected to the switch therapy experimental protocol at test-of-cure visit (4-6 weeks after the last dose of ciprofloxacin) and at follow-up visit (six months after the end of therapy)

\begin{tabular}{|c|c|c|c|c|c|c|}
\hline \multirow[t]{3}{*}{ Microorganism } & \multicolumn{6}{|c|}{ Number of patients } \\
\hline & \multicolumn{3}{|c|}{ Test-of-cure visit } & \multicolumn{3}{|c|}{ Follow-up visit } \\
\hline & $\mathbf{E}$ & $\mathbf{P}$ & ES/P & $\mathbf{E}$ & $\mathbf{P}$ & ES/P \\
\hline Enterococcus faecalis & 8 & 1 & 0 & 8 & 0 & 1 \\
\hline Escherichia coli & 7 & 1 & 0 & 6 & 1 & 1 \\
\hline Proteus mirabilis & 3 & 0 & 0 & 3 & 0 & 0 \\
\hline Klebsiella pneumoniae & 2 & 1 & 0 & 3 & 0 & 0 \\
\hline Citrobacter spp. & 2 & 0 & 0 & 2 & 0 & 0 \\
\hline Enterobacter spp. & 2 & 0 & 0 & 2 & 0 & 0 \\
\hline Pseudomonas aeruginosa & 1 & 0 & 0 & 0 & 0 & 1 \\
\hline E. coli + E. faecalis & 1 & 0 & 0 & 0 & 0 & 1 \\
\hline$P$. aeruginosa $+E$. faecalis & 1 & 0 & 0 & 0 & 0 & 1 \\
\hline Total & $27(90 \%)$ & $3(10 \%)$ & - & $24(80 \%)$ & $1(3,3 \%)$ & $5(16,7 \%)$ \\
\hline
\end{tabular}

$E$ - eradication; $P$ - persistence; $E S / P$ - eradication with superinfection, (or persistence following apparent eradication) 
ative pathogens at the TOC visit, and 3 showed microbiological persistence. Six months after the end of therapy bacterial infection was detected in 6 patients. In one case the same baseline pathogen type (E. coli) persisted throughout the whole study. In two cases eradication of the baseline pathogen with superinfection by a different organism was observed, whereas in three cases baseline pathogens, apparently eradicated at the TOC visit, were detected during the follow-up visit.

All enrolled patients had severe symptoms at baseline as inclusion criterion. NIH-CPSI pain + voiding scores ranged between 19 and 29, and total scores ranged between 25 and 38. The antibacterial switch-therapy protocol caused a marked attenuation of CBP clinical symptoms, measured with the $\mathrm{NIH}$-chronic prostatitis symptom index. At the TOC visit, 25 patients (83\%) showed mild or absent symptoms, whereas 5 patients (17\%) showed persistence of severe symptoms (Fig. 1). No patient had moderate symptoms. At the 6-month follow-up visit, two more patients showed severe symptoms (23\%), whereas the remaining 23 persisted in a condition of symptom remission (Fig. 1). The decrease of pain + voiding scores, of quality of life impact scores and of NIH-CPSI total scores in the entire patient cohort was statistically significant in all cases $(p<0.01$, baseline vs. TOC scores; $p<0.01$, baseline vs. follow-up scores, Wilcoxon signed rank test).

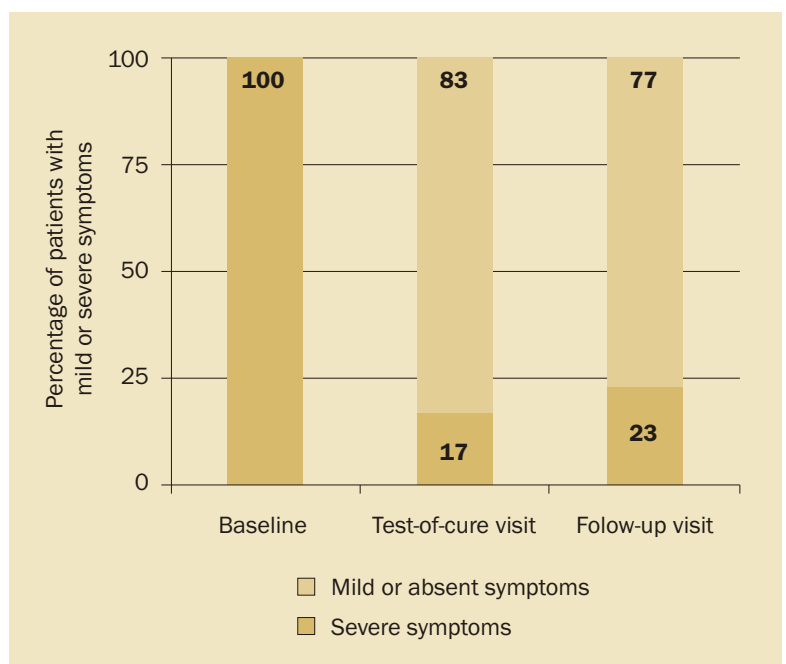

Figure 1. Clinical efficacy of a 4-week switch-therapy protocol based on administration of intravenous azithromycin (500 $m g$ once-daily) and ciprofloxacin (400 mg twice-daily) for three days, followed by oral ciprofloxacin $(500 \mathrm{mg}$ twicedaily) for 25 days. The NIH-CPSI questionnaire was administered to 30 patients at enrolment, at the test-of-cure visit (4-6 weeks after the last ciprofloxacin dose), and at the follow-up visit, 6 months after the end of therapy. Clinical symptoms are presented as the sum of pain and voiding symptom scores. According to McNaughton Collins et al.,14 symptoms were classified as mild (0-9 points), moderate (10-16 points) and severe (17-31 points). Patients of this cohort did not show moderate symptoms at any time-
We performed post-hoc "compromise" power analysis ${ }^{18}$ to assess whether statistical inference about clinical improvement scores was characterized by adequate power. We computed the achieved power given $\alpha=0.05$, a sample size of 30 subjects, $q=\beta / \alpha=4$, and effect sizes of (i) 2.57 and 2.22 calculated for the comparison of baseline $\mathrm{NIH}$ CPSI total scores with TOC and follow-up data, respectively, (ii) 2.35 and 2.17, for the comparison of baseline NIHCPSI clinical symptoms (pain + voiding) scores with TOC and follow-up data, respectively, and (iii) 1.81 and 1.68, for the comparison of baseline NIH-CPSI quality of life impact scores with TOC and follow-up data, respectively. Comparisons were shown to be adequately powered (1$\beta>0,99$ in all cases).

\section{Discussion}

Intravenous administration of antibacterial agents is currently reserved for cases of acute bacterial prostatitis. International guidelines recommend parenteral administration of high doses of bactericidal antibiotics, such as broad-spectrum penicillins, third-generation cephalosporins or fluoroquinolones, combined or not with an aminoglycoside. ${ }^{3,4,5}$ After defervescence and normalization of infection parameters, treatment can be switched to oral antibiotics, and continued for about 2-4 weeks. ${ }^{3,4,5}$

The present pilot study was aimed at assessing whether a switch-therapy regime could also be suitable for severe cases of chronic bacterial prostatitis. The results presented in this paper demonstrated the efficacy and safety of a 4-week therapy protocol based on the intravenous administration of ciprofloxacin and azithromycin, followed by oral ciprofloxacin. Microbiological eradication was achieved in $90 \%$ of patients (27 out of total 30 enrolled subjects). This effect was associated with prompt resolution of severe CBP symptoms, assessed with the NIH-CPSI test. In three cases of multiple infection, apparent microbiological eradication at test-of-cure visit was followed by reappearance of the same pathogens assessed at baseline. Interestingly, in all other cases, no infection relapse has been reported to this day (about 12 months off-therapy). The most likely reasons for relapse of infection in CBP are the relatively low prostatic concentrations of antibiotics achievable with currently available oral agents and dosages, or the persistence of pathogens within the prostate gland in the form of quorum-sensing regulated biofilms. ${ }^{19}$ Compared to planctonic pathogens, sessile bacterial biofilms are characterized by different metabolic patterns and by reduced susceptibility to antibacterial agents. The administration of agents able to dissolve or inhibit biofilms, or the achievement of increased serum and, in turn, tissue concentrations of antibiotics are two conceivable therapeutic approaches to relapsing CBP. Both approaches were attempted by administering fluoroquinolones and macrolides intravenously.

Macrolide antibiotics do not only provide clinical benefit in chronic bacterial infections as a result of direct antibacterial effects. They exhibit biofilm inhibiting effects, as well 
as a variety of immunomodulatory actions which promote host defense. ${ }^{20-24}$ These effects of macrolides are well known and extensively exploited in the treatment of pulmonary conditions. In cystic fibrosis and bronchiolitis obliterans, combined actions of macrolides probably result in increased clinical efficacy than their direct antibacterial actions alone. ${ }^{25}$

Azithromycin is an inhibitor of the generation of auto-inducing, quorum-sensing homoserine lactone molecules that regulate bacterial growth and biofilm production, ${ }^{22,23}$ and can also prevent the production of alginate, the most prominent component of biofilm, via inhibition of GDPmannose dehydrogenase. In addition, azithromycin can stimulate neutrophils, thereby promoting acute antibacterial defense, but subsequently inhibits several neutrophilsynthesized inflammatory chemokines and cytokines ${ }^{20,22}$ and promotes neutrophil apoptosis, which helps to resolve inflammation. ${ }^{26,27}$ In summary, these pleiotrophic effects of azithromycin, combined with their intrinsic antibacterial properties and with the bactericidal activity of ciprofloxacin may have concurred to the high eradication rates observed in our patient cohort.

When administered orally, azithromycin shows $~ 40 \%$ bioavailability, due to incomplete drug absorption. ${ }^{28}$ Following single oral doses of $500 \mathrm{mg}$ azithromycin, Cmax and AUC24 were reported to be $0.41 \mu \mathrm{g} / \mathrm{ml}$ and $2.6 \mu \mathrm{g} \cdot \mathrm{h} / \mathrm{ml}$, respectively. These values are approximately $38 \%$ and $52 \%$ of the values observed following a single $500 \mathrm{mg}$ 3-hour intravenous infusion (Cmax: $1.08 \mu \mathrm{g} / \mathrm{ml}$, AUC24: $5.0 \mu \mathrm{g} \cdot \mathrm{h} / \mathrm{ml}){ }^{29}$

Increased bioavailability and higher peak plasma concentrations achieved upon intravenous administration of azithromycin may also have concurred to the favorable microbiological and clinical results of treatment.
It is important to emphasize that azithromycin shows unique pharmacokinetic/pharmacodynamic (PK/PD) properties, compared to other macrolide antibiotics. Whereas erythromycin shows a time-dependent antibacterial activity, the AUC24/MIC is the primary factor influencing the clinical efficacy of azithromycin. ${ }^{30,31}$ Therefore, azithromycin behaves like ciprofloxacin, which shows a concentration-dependent killing activity, and was co-administered with the macrolide.

In conclusion, elevated peak plasma concentrations achieved with intravenous administration may possibly lead to increased prostatic concentrations of both azithromycin and ciprofloxacin, and ultimately to enhanced antibacterial, antibiofilm and anti-inflammatory activity of the tested drug combination. As no adverse effects were recorded or spontaneously reported by patients, increased drug concentrations apparently did not cause the emergence of signs of toxicity. Due to the noncomparative design of our study, these results should be evaluated conservatively and confirmed in a randomized controlled setting.

\section{Acknowledgements}

Research and publication expenses were supported in part by the National Foundation for Science, Higher Education and Technological Development of the Republic of Croatia (project no. 04/30 "Research on the etiology, epidemiology, diagnostics and treatment of patients with prostatitis syndrome") and PLIVA Croatia Ltd.

We thank Ms Arijana Pavelić for her help in the preparation of the manuscript.

\section{References}

1. WENNINGER K, HEIMAN JR, ROTHMAN I, BERGHUIS JP, BERGER RE. Sickness impact of chronic nonbacterial prostatitis and its correlates. J Urol 1996; 155 (3): 965-68.

2. KRIEGER JN, NYBERG L JR, NICKEL JC. NIH consensus definition and classification of prostatitis. JAMA 1999; 282: 236-37.

3. WAGENLEHNER FM, WEIDNER W, NABER KG. Therapy for prostatitis, with emphasis on bacterial prostatitis. Exp opin pharmacother 2007;8(11):1667-74.
4. MAGRI V, PERLETTI G, BARTOLETTI R, CAI T, EMELYANOVA I, MEHIK A, et al. Critical issues in chronic prostatitis. Arch Ital Urol Androl 2010; 82 (2):75-82.

5. European Association of Urology Urological Infections Guidelines: http://www.uroweb.org/gls/pdf/15_Urological_ Infections.pdf

6. WAGENLEHNER FM, NABER KG, BSCHLEIPFER T, BRÄHLER E, WEIDNER W. Prostatitis and male pelvic pain syndrome: diagnosis and treatment. Dtsch Arztebl Int 2009; 106 (11): 175-83. 
7. NABER KG, European Lomefloxacin Prostatitis Study Group. Lomefloxacin versus ciprofloxacin in the treatment of chronic bacterial prostatitis. Int J Antimicrob Agents 2002; 20 (1): 18-27.

8. PAGLIA M, PETERSON J, FISHER AC, QIN Z, NICHOLSON SC, KAHN JB. Safety and efficacy of levofloxacin $750 \mathrm{mg}$ for 2 weeks or 3 weeks compared with levofloxacin 500 mg for 4 weeks in treating chronic bacterial prostatitis. Curr Med Res Opin 2010; 26 (6): 1433-41.

9. MAGRI V, MONTANARI E, ŠKERK V, MARKOTIĆ A, MARRAS E, RESTELLI A, et al. Fluoroquinolone-macrolide combination therapy for chronic bacterial prostatitis: retrospective analysis of pathogen eradication rates, inflammatory findings and sexual dysfunction. Asian J Androl 2011, in press, doi: 10.1038/aja.2011.36

10. MAGRI V, TRINCHIERI A, POZZI G, RESTELLI A, GARLAS$\mathrm{CHI} M C$, TORRESANI E, et al. Efficacy of repeated cycles of combination therapy for the eradication of infecting organisms in chronic bacterial prostatitis. Int J Antimicrob Agents 2007; 29 (5): 549-56.

11. MAGRI V, TRINCHIERI A, CERIANI I, MARRAS E, PERLETTI G. Eradication of unusual pathogens by combination pharmacological therapy is paralleled by improvement of signs and symptoms of chronic prostatitis syndrome. Arch Ital Urol Androl 2007; 79 (2): 93-8 .

12. MAGRI V, TRINCHIERI A, MONTANARI E, DEL NERO A, MANGIAROTTI B, ZIRPOLI P, et al. Reduction of PSA values by combination pharmacological therapy in patients with chronic prostatitis: implications for prostate cancer detection. Arch Ital Urol Androl 2007; 79 (2): 84-92.

13. MAGRI V, MARRAS E, ŠKERK V, MARKOTIĆ A, RESTELLI A, GARLASCHI MC, PERLETTI G. Eradication of Chlamydia trachomatis parallels symptom regression in chronic bacterial prostatitis patients treated with a fluoroquinolonemacrolide combination. Andrologia 2010; 42 (6): 366-75.

14. M'NAUGHTON COLLINS M, PONTARI MA, O'LEARY MP, CALHOUN EA, SANTANNA J, LANDIS JR, et al. Quality of life is impaired in men with chronic prostatitis: the Chronic Prostatitis Collaborative Research Network. J Gen Intern Med 2001; 16 (10): 656-62.

15. STAMEY TA. Prostatitis. J R Soc Med 1981; 74: 22-40

16. PROPERT KJ, LITWIN MS, WANG Y, ALEXANDER RB, CALHOUN E, NICKEL JC, et al. Responsiveness of the National Institutes of Health Chronic Prostatitis Symptom Index (NIH-CPSI). Qual Life Res 2006; 15 (2): 299-305.

17. FAUL F, ERDFELDER E, BUCHNER A, LANG AG. Statistical power analyses using G*Power 3.1: Tests for correlation and regression analyses. Behav Res Meth 2009; 41: 1149-60.

18. FAUL F, ERDFELDER E, LANG AG, BUCHNER A. G*Power 3: A flexible statistical power analysis program for the social, behavioral, and biomedical sciences. Behav Res Meth 2007; 39: 175-91.
19. MAZZOLI S. Biofilms in chronic bacterial prostatitis (NIHII) and in prostatic calcifications. FEMS Immunol Med Microbiol 2010; 59 (3): 337-44.

20. ČULIĆ O, ERAKOVIĆ V, PARNHAM MJ. Anti-inflammatory effects of macrolide antibiotics. Eur J Pharmacol 2001; 429: 209-29.

21. PARNHAM MJ. Immunomodulatory effects of antimicrobials in the therapy of respiratory tract infections. Curr Opinion Infect Dis 2005; 18: 125-31.

22. TATEDA K, ISHII Y, KIMURA S, HORIKAWA M, MIYAIRI S, YAMAGUCHI K. Suppression of Pseudomonas aeruginosa quorum-sensing systems by macrolides: a promising strategy or an oriental mystery? J Infect Chemother 2007; 13: 357-67.

23. KANOH S, RUBIN BK. Mechanisms of action and clinical application of macrolides as immunomodulatory medications. Clin Microbiol Rev 2010; 23: 590-15.

24. GIAMARELLOS-BOURBOULIS EJ. Macrolides beyond the conventional antimicrobials: a class of potent immunomodulators. Int J Antimicrob Agents 2008;31 (1): 12-20

25. SCHULTZ MJ. Macrolide activities beyond their antimicrobial effects: macrolides in diffuse panbronchiolitis and cystic fibrosis. J Antimicrob Chemother 2004; 54 (1): 21-8.

26. ČULIĆ O, ERAKOVIĆ V, ČEPELAK I, BARIŠIĆ K, BRAJŠA K, FERENČIĆ Ž, et al. Azithromycin modulates neutrophil function and circulating inflammatory mediators in healthy human subjects. Eur J Pharmacol 2002; 450: 277$-89$.

27. SILVA MT. Macrophage phagocytosis of neutrophils at inflammatory/infectious foci: a cooperative mechanism in the control of infection and infectious inflammation. J Leuk Biol 2011; 89: 675-83.

28. BERINGER P, HUYNH KM, KRIENGKAUYKIAT J, BI L, HOEM N, LOUIE S, HAN E, et al. Absolute bioavailability and intracellular pharmacokinetics of azithromycin in patients with cystic fibrosis. Antimicrob Agents Chemother 2005; 49 (12): 5013-17.

29. Technical document on azithromycin (Teva Parenteral Medicines, Inc) NIH DAILYMED Archive, http://dailymed. nlm.nih.gov/dailymed/lookup.cfm?setid=0e76db5f-3f274c92-b270-ff4671b77a7a

30. NIGHTINGALE CH. Pharmacokinetics and pharmacodynamics of newer macrolides. Pediatr Infect Dis J 1997; 16 (4): 438-43.

31. VAN BAMBEKE F, TULKENS PM. Macrolides: pharmacokinetics and pharmacodynamics. Int J Antimicrob Agents 2001; 18: S17-S23.

\section{Adresa za dopisivanje:}

Prof. dr. sc. Višnja Škerk, dr. med.

Klinika za infektivne bolesti "Dr. Fran Mihaljević" 10000 Zagreb, Mirogojska 8

e-mail: visnja.skerk@bfm.hr

DOUBLE PUBLICATION

with permission from

J CHEMOTHER

2011,23(6):350-3. 